\title{
Obstacle Detectability of Ultrasonic Ranging System and Sonar Map Understanding
}

\author{
Akihisa OHYA, Takayuki OHNO and Shin'ichi YUTA \\ Intelligent Robot Laboratory \\ Institute of Information Sciences and Electronics, University of Tsukuba \\ Tsukuba 305, JAPAN \\ Phone: +81-298-53-5155 Fax: +81-298-53-5206 \\ E-mail: ohya@roboken.is.tsukuba.ac.jp
}

\begin{abstract}
Information obtained by the ultrasonic sensor is influenced by the characteristics of the sensing system such as sensitivity, directivity and so on. In order to investigate its influence, we constructed two ultrasonic ranging systems of which characteristics differs from each other and examined their performance such as obstacle detectability and resultant sonar map.
\end{abstract}

Keywords: Ultrasonic Sensing, Obstacle Detection, Sonar Map

\section{INTRODUCTION}

For mobile robots, functions which recognize environments are required to find unpredictable obstacles and paths through which the robot can pass, whether having an environmental map or not. As for range sensors, which can measure a distance to objects, ultrasonic sensor is more commonly used with mobile robots because it is small, inexpensive and easy to calculate distances.

Present ultrasonic sensor systems generally calculate distance using the time-of-flight (TOF) method. The distance $l$ to a reflected object is calculated by

$$
l=\frac{c t}{2},
$$

where $c$ is the speed of sound, and $t$ is the round-trip time-of-flight (Fig. 1). The TOF method produces a range value when the echo amplitude first exceeds the threshold level after transmitting. In spite of the simple method like this, information obtained by the ultrasonic sensor is influenced by the characteristics of the sensing system, it of environment and so on.

In this paper, in order to investigate the influence of the sensor system, we constructed two ultrasonic ranging systems of which characteristics differs from each other and examined their performance such as obstacle detectability and resultant sonar map.

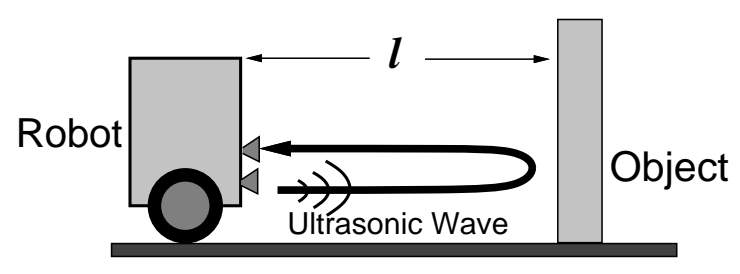

Fig. 1. The principles of the time-of-flight (TOF) method. 
In section II, we introduce two ultrasonic ranging systems which we developed. Their availability for obstacle detection is examined in section III, sonar map making using them in section IV. Finally, the conclusions are presented in section V.

\section{Two Ultrasonic Ranging Systems}

Fig. 2 shows a model of reflected waves, where there are two objects in a field of view. As an ultrasonic wave attenuates and spreads, the echo amplitude reflected off farther object is smaller (even from the same object[2]). Because we use piezoelectric ultrasonic sensors, we use a transmitter and a receiver separately. So, the received waves include the direct wave from the transmitter which must be neglected.

We have developed a ultrasonic range finding system $\mathrm{A}$ in which the following fundamental method is employed[4][3]. Ultrasonic waves are discharged from a transmitter given comparatively long burst waves in order to vibrate its piezoelectric vibrator fully. A range value is calculated by TOF method using the amplified echo and a threshold level. The threshold level to detect echoes is constant in order to simplify the circuit. The concept of each signal for System A is shown in Fig. 3. However, this system has some problems. First, the rise of echo signal is slow, since the intensity of the echo signal is not so large. So measuring errors result. To reduce such measuring errors, the rise of ultrasonic waves must be quickened. Next, the measuring range is limited by a fixed threshold level. It is impossible to detect far distance objects when the level is set higher, or it is likely to detect noise from near distance objects when the level is set lower. Also, horns are attached to System A to increase the intensity of waves. However, it makes the directivity narrow.

To solve the problems of System A, we improved the transmit and receive circuit, and developed a new ultrasonic sensor system B. The concept of each signal for System B is shown in Fig. 4. In order to enlarge the intensity of echo signal and quicken the rise of it, a high voltage single pulse is employed for transmitting. The peak voltage is about $720 \mathrm{~V}$, while it is about $12 \mathrm{~V}$ in System A. This method has the following benefits. First, the masking time can be shortened by shortening the transmitting time. Thus it is possible to measure near distances. Second, the rise time of transmitting waves is shortened by using a high voltage for discharging ultrasonic pulses. Therefore the measuring errors can

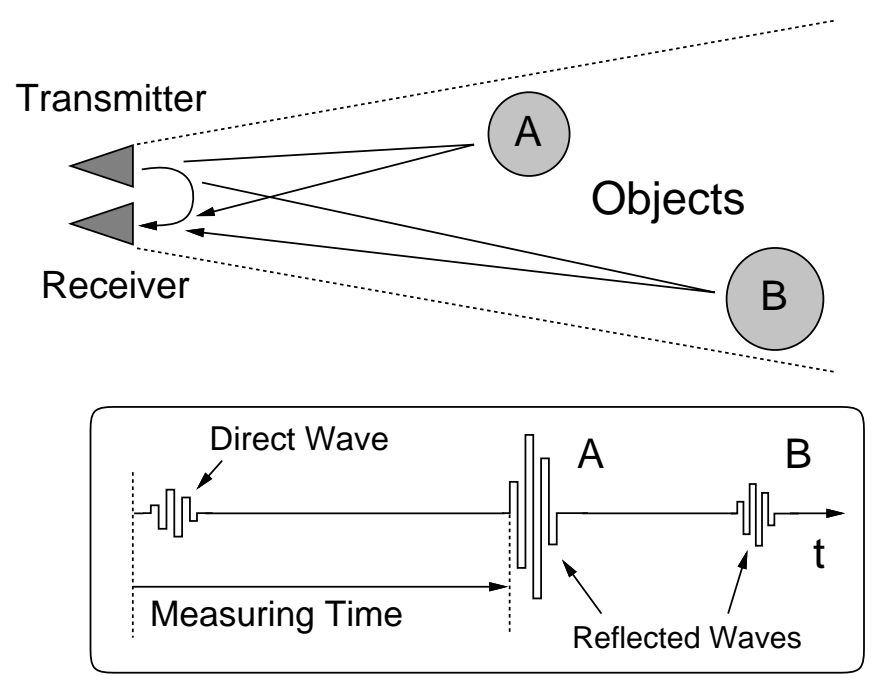

Fig. 2. A model of reflected waves. 
be reduced. For receiving, the threshold level is decreased with time, and is gradually adjusted to the echo amplitude decreasing with the distance. We call this method timethreshold-control. This method is robust to noises from close objects, and makes it possible to measure far distance object. The Polaroid ultrasonic range sensor[1] solves this problem by changing the amplification factor with time. This is called time-gain-control. But the circuit is complicated. To solve this problem with a simple circuit, not the amplification factor but the threshold level should be varied with time. System B realizes increases in the measurable range, decreases measuring errors, and increases measuring performance with a simple circuit.

Fig. 5 shows an example of echo signal obtained by System A. The left one is a direct wave, and the right is an echo. In this case, if ignoring the direct wave, it was impossible to measure near objects whose echo returns in this time. Also, measuring errors resulted because of slow rise of ultrasonic wave. Fig. 6 shows an example of echo signal obtained by System B. It shows that the direct wave is shortened, and the rise time of ultrasonic waves is also shortened. Fig. 7 shows the amplified received signal and the threshold level in System B. The received waves are saturated around $4 \mathrm{~V}$. The direct wave is ignored by making threshold level high at first. After that, the threshold level is decreased with time.

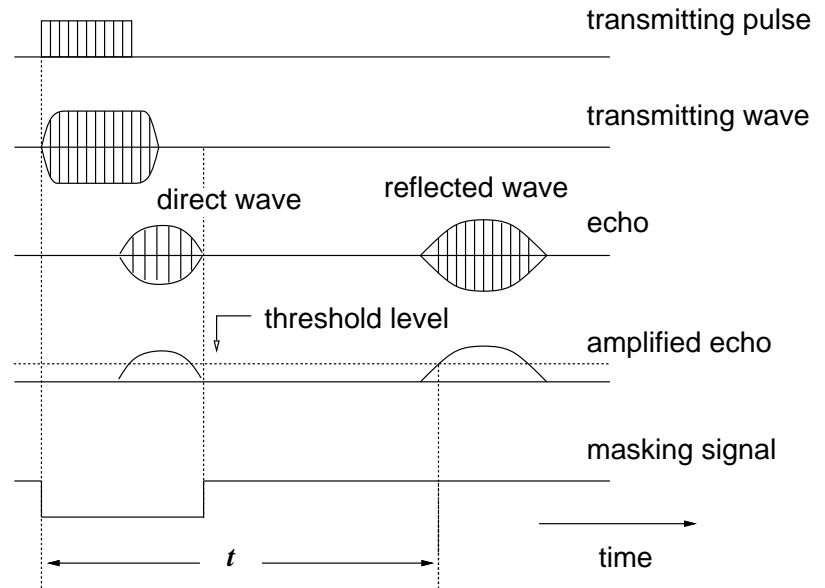

Fig. 3. The concept of each signal for System A.

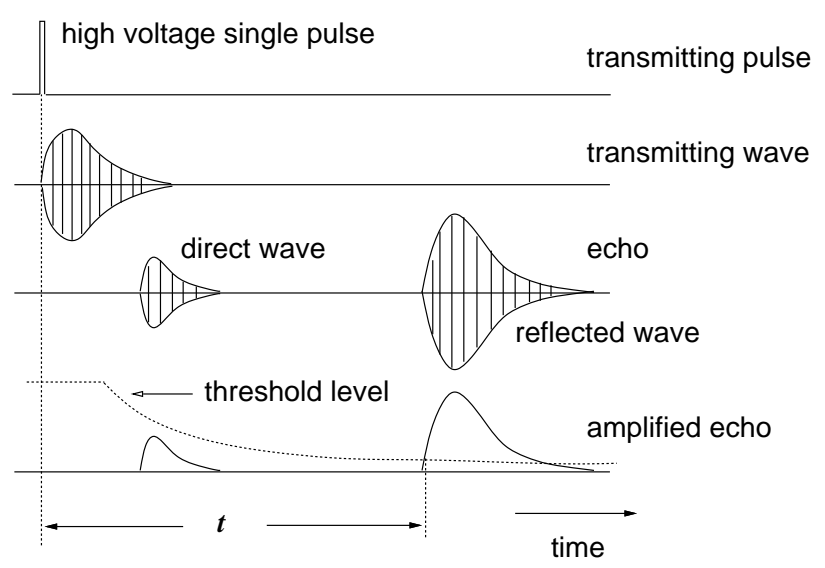

Fig. 4. The concept of each signal for System B. 


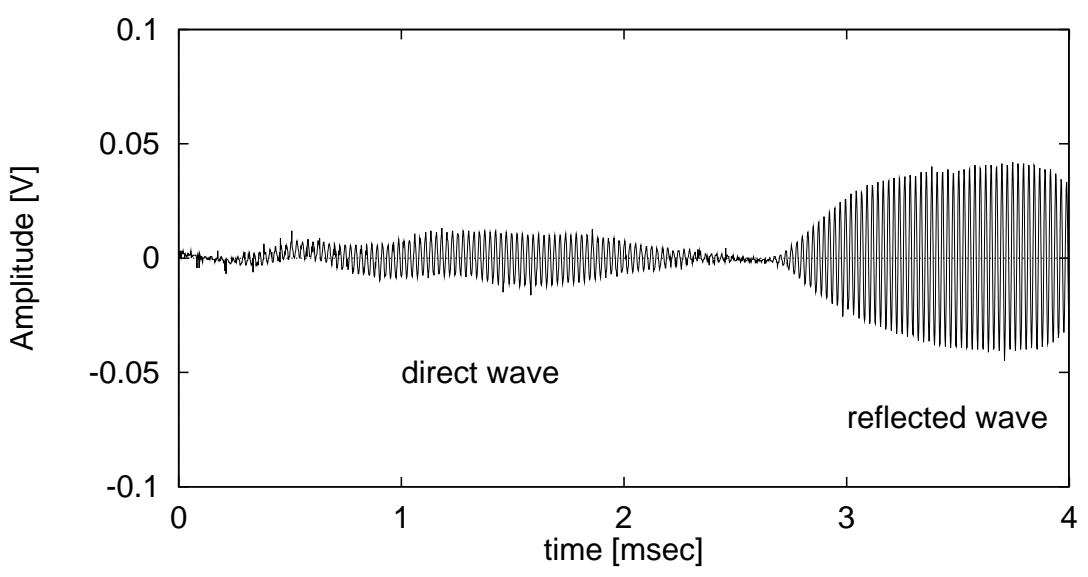

Fig. 5. An example of echo signal obtained by System A.

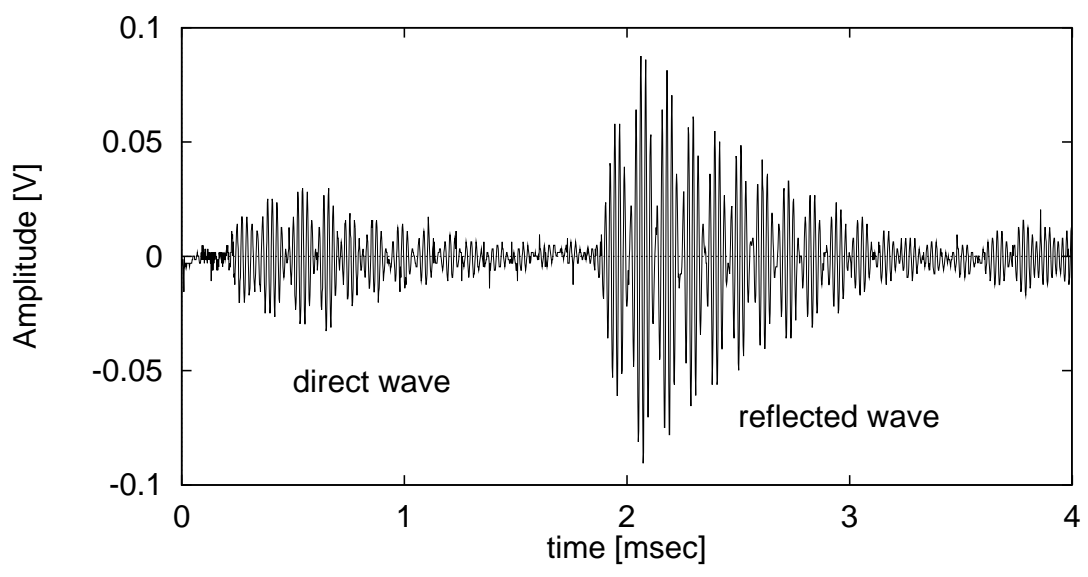

Fig. 6. An example of echo signal obtained by System B.

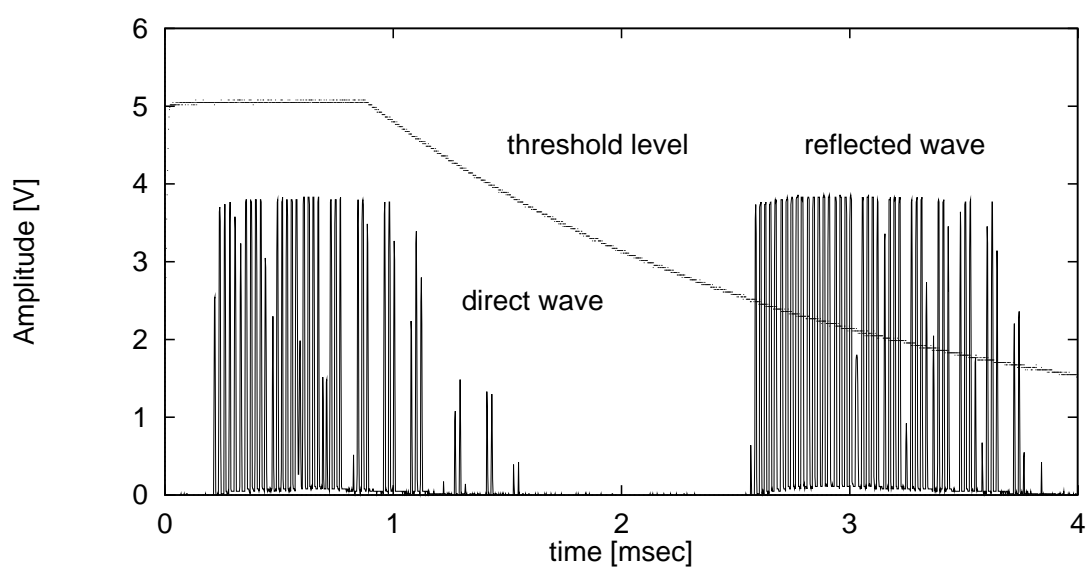

Fig. 7. An example of the amplified received signal and the threshold level in System B. 


\section{Obstacle Detectability}

To examine the availability for obstacle detection of two ultrasonic ranging systems mentioned in previous section, we measured the relation of the maximum measurable distance to the width of a reflected object. The conventional system is attached to a horn to increase the intensity of echoes. We measured the maximum range data to the object which can be detected, and errors to the real distance. The experimental set-up is shown in Fig. 8, and the experimental results are shown in Fig. 9. In Fig. 9, the result of System A is below the graph, and System B is above. Solid lines mean the measured value, and dotted lines mean the real distance to the object. As shown in this figure, in both System A and B, the maximum measurable distances decrease steeply if the width of the reflected object is smaller than $10 \mathrm{~cm}$. However, this figure indicates that System B can measure farther distances than System A, without using horn in System B. Also the errors of System B to the real distance are smaller than that of System A, we conclude the reliability and the measuring performance are improved with System B.

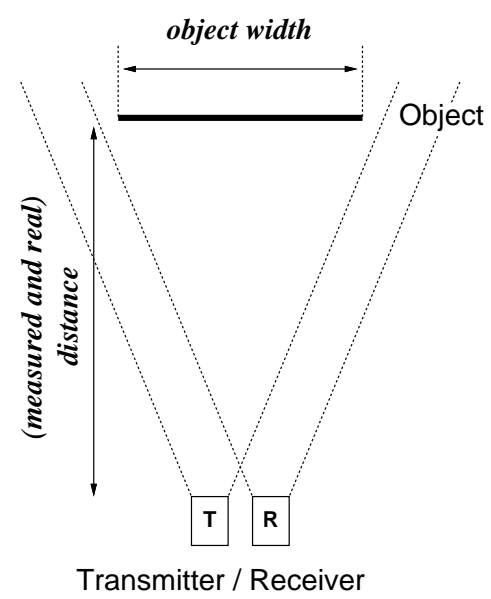

Fig. 8. Experimental set-up for the measurement of the relation of the maximum measurable distance to the width of a reflected object.

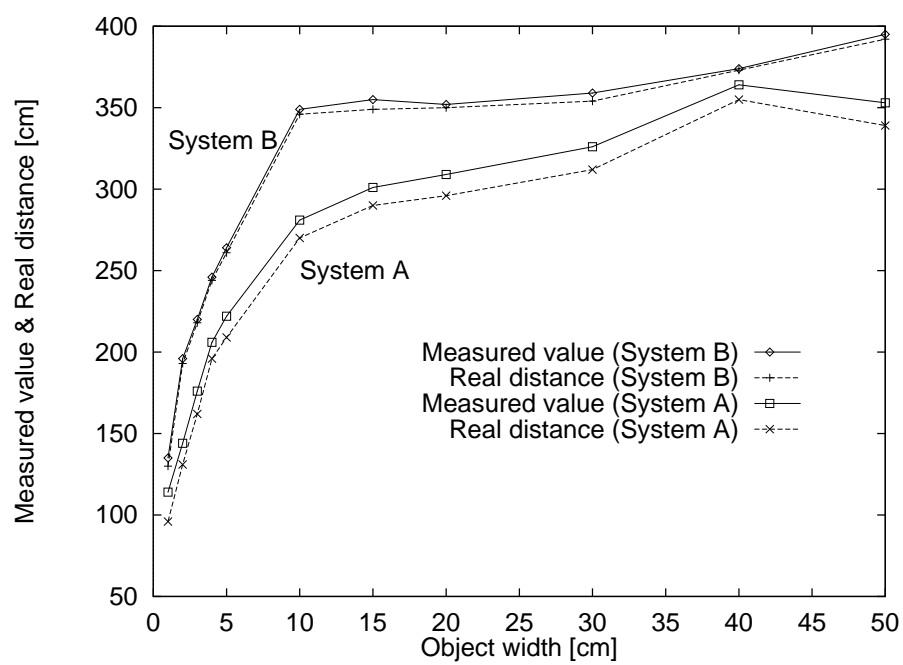

Fig. 9. The change of the maximum measurable distance to the width of a reflected object. 


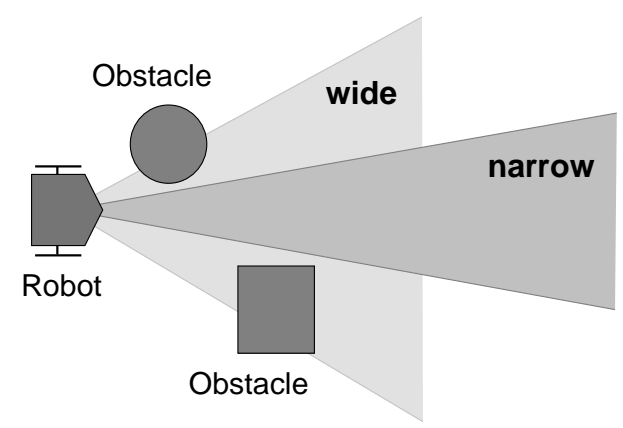

Fig. 10. The directivity of ultrasonic sensor for the obstacle detection. The obstacles cannot be detected by the narrow directivity.

In many cases, ultrasonic sensors are attached to a horn to increase the intensity of waves on the transducer line-of-sight, so the directivity becomes narrow. A narrow directivity is better in order to know the exact direction which obstacles exist. However, only obstacles which face perpendicularly the transducers line-of-sight can be detected with a narrow directivity. It is important for mobile robots' obstacle detection to know whether obstacles exist or not, and how far there are. So, a wide directivity is also required for obstacle detection as shown in Fig. 10. With System B, more sensitivity can be obtained without a horn than with System A. Therefore the directivity of System B can be wide as is verified in next section, and it is more available for obstacle detection.

\section{Sonar Map Understanding}

We investigate environment map making using two ultrasonic ranging systems. The method of making maps was for the range data to be placed along the transducer orientation at a measured range. There were flat walls and right-angle corners (convex and

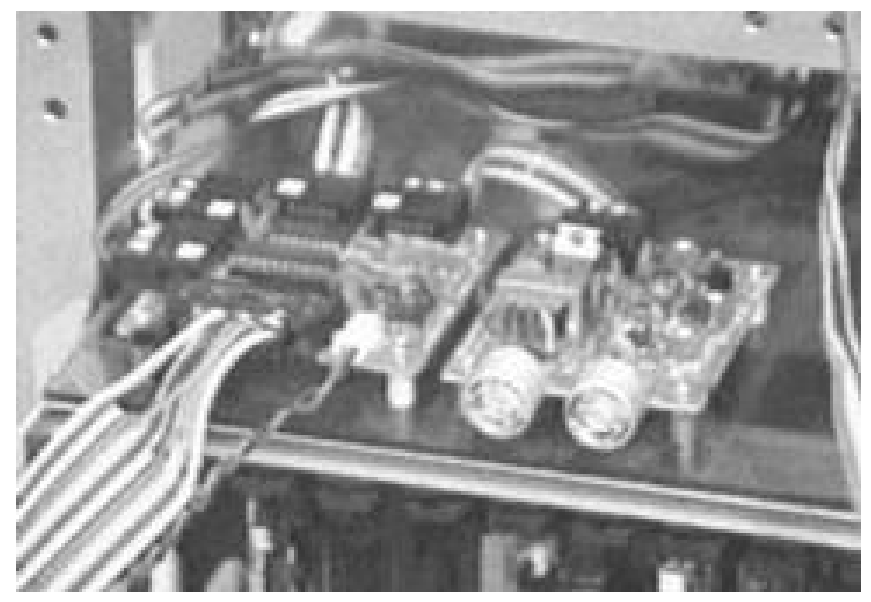

Fig. 11. System B mounted on our mobile robot "YAMABICO". The right side is a transmit and receive circuit for one direction $(70 \mathrm{~mm} \times 60 \mathrm{~mm})$, the left side is a high voltage power supply for 4 transmit and receive circuits $(70 \mathrm{~mm} \times 72 \mathrm{~mm})$. Transducers are attached to the board directly, without horns. 
concave). System A used a horn to increase its intensity. Each system is mounted on our mobile robot "YAMABICO"[5] (Fig. 11), and the orientation of the system was changed by rotating the robot. The experimental environment is shown in Fig. 12, the experimental results of System A and B are shown in Fig. 13 and 14, respectively. The sensors were put on the origin $(0,0)$.

As shown in Fig. 13, System A detected only echoes from which the transducer line-ofsight is perpendicular to the wall, because the intensity of echo signal is low in this system and the echo amplitude cannot exceed a threshold level when the echo is reflected out of transducer line-of-sight. As a result, it seems this system has a narrow directivity. Also, the intensity of echoes becomes smaller as the angle between the transducer line-of-sight and the normal direction of the wall becomes larger. Then, the echo amplitude exceeds later a threshold level. Consequently, the range data lay on the arc of a circle which was contact with the wall, and its center was out of walls.

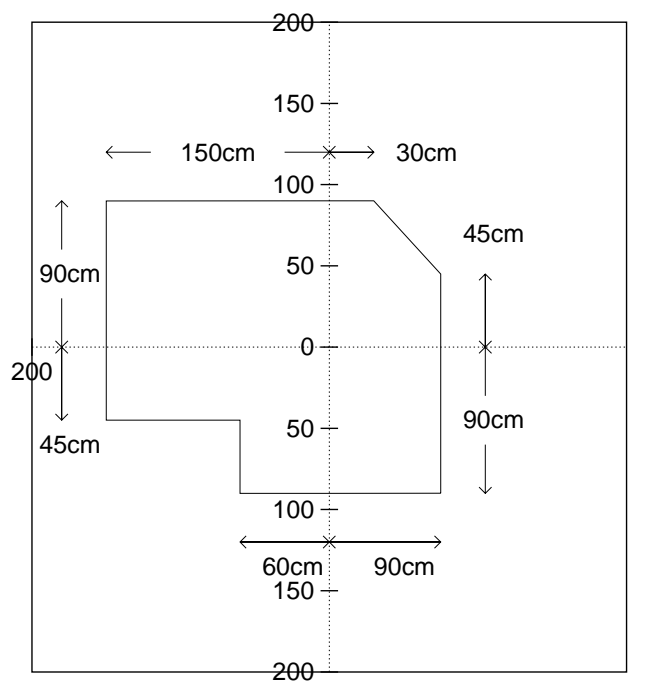

Fig. 12. Experimental environment. Sensors were put at the point 0. 
Compared with above, Fig. 14 indicates that System B could detect echoes in every direction, because the intensity of echo signal is large enough in this system. It can be seen from this figure that the directivity of System B is wide, since the range data lay on the arc of a circle with center at the sensor. Therefore, when a robot moves, obstacles can be detected even if they have no perpendicular faces to transducer line-ofsight. Consequently, the ability to detect obstacles is improved in System B.

\section{CONClusion}

It is concluded from the above-mentioned results that:

- Obstacle detectability varys with the individual sensor system.

- The directivity of the sensor depends on not only directivity of the transducer but sensitivity of sensor.

- The shape of the resultant sonar map changes drastically according to the characteristics of the sensor such as sensitivity, directivity and so on.

- Understanding of the sonar map should be done with carefulness, because the shape of sonar map may be largely different form the real shape of the environment.

If the sensing ability of robot is nearly equal to it of human, we can trust the robot. Unfortunately, almost current sensors are not so intelligent and the ability is limited. So, we must be careful in the presence of the robot.

\section{REFERENCES}

[1] Polaroid Corp., Cambridge, Massachusetts 02139, Ultrasonic Ranging System.

[2] P.J. McKerrow and J.C.T. Hallam: "An Introduction to the Physics of Echolocation," Proceedings of the Third National Conference on Robotics of the Australian Robot Association, pp.198-209, Melbourne, June 1990.

[3] Yoshiaki Nagashima and Shin'ich Yuta: "Ultrasonic Sensing for a Mobile Robot to Recognize an Environment -Measuring the Normal Direction of Walls-," Proceedings of the 1992 IEEE/RSJ International Conference on Intelligent Robots and Systems, pp.805-812, Raleigh, NC, July 1992.

[4] Minho Song and Shin'ichi Yuta: "Autonomous Mobile Robot Yamabico and Its Ultrasonic Range Finding Module," '89 Korean Automatic Control Conference, Vol.2, pp.711-714, Seoul, October 1989.

[5] Shin'ichi Yuta, Sho'ji Suzuki and Shigeki Iida: "Implementation of a Small Size Experimental Self-contained Autonomous Robot -Sensors, Vehicle Control and Description of Sensor Based Behavior-," R. Chatila et al. Eds, Experimental Robotics II (The 2nd International Symposium on Experimental Robotics, Toulouse, June 1991), SpringarVerlag, pp.344-359, 1993. 


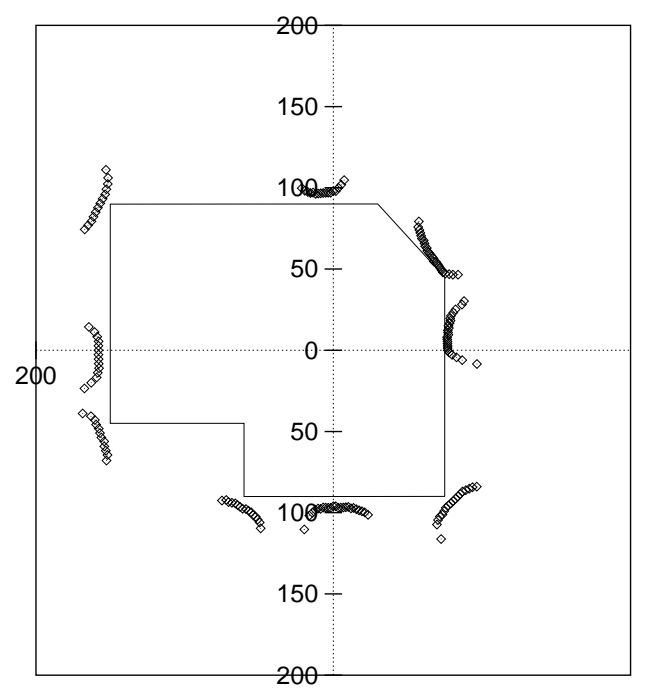

Fig. 13. Resultant sonar map for System A.

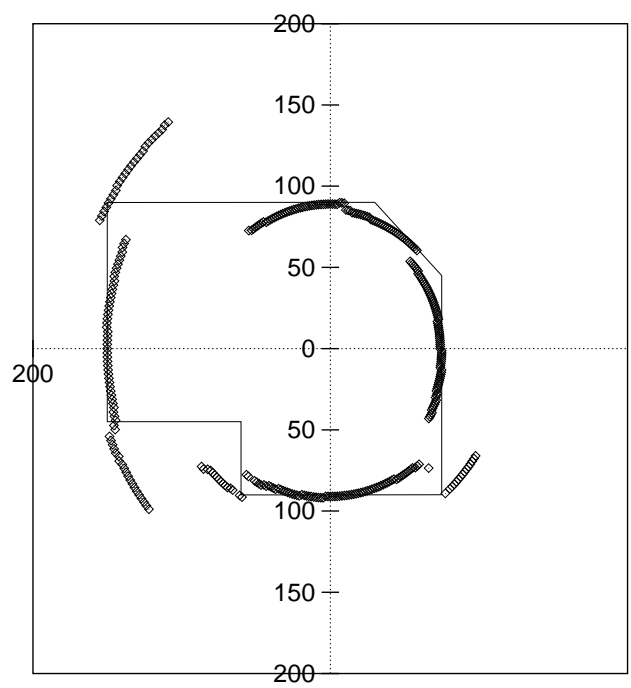

Fig. 14. Resultant sonar map for System B. 